\title{
0 uso de caixas excito-repelentes com Anopheles darlingi Root, A. nuneztovari Gabaldon e Culex pipiens quinquefasciatus Say obtidos em áreas perto de Manaus, Amazonas
}

\author{
J. Derek Charlwood (*) \\ Norivai D. Paraluppi (*)
}

\begin{abstract}
Resumo
Caixas contendo DDT foram usadas para realizar testes de excito-repelência em populações de Anopheles darlingi, A nuneztovari e Culex pipiens quinquefasciatus nas proximidades da cidade de Manaus, Amazonas. As duas espécies de anofelinos mostraram uma fuga rápida do DDT, enquanto que a população de Culex apresentou uma alta resistência, sendo pouco influenciada pela presença do inseticida. Foram usadas também caixas como armadilhas de repouso para os Culex, e neste caso houve pouca diferença entre a caixa com DDT e a de controle. Discute-se a necessidade de padronizar testes de excito-repelência para elucidar o conceito desta resistência comportamental.
\end{abstract}

\section{INTRODUÇÃO}

$\mathrm{O}$ uso do DDT como um agente efetivo contra os mosquitos vetores de doenças tem sido influenciado pelo desenvolvimento de uma resistência da população exposta ao inseticida, e por respostas comportamentais do inseto, que tendem a reduzir seu contato com o inseticida (Schoof, 1970). O DDT produz um efeito irritante, notável na maioria dos mosquitos. Isto os obriga a voar para longe da superfície borrifada, antes que absorvam uma dose tóxica do mesmo. O efeito irritante se manifesta não somente no contato com a superfície borrifada, mas também produz um eteito repelente nos mosquitos (WHO 1975). Em geral, as populações susceptíveis são mais irritáveis pelo DDT do que as populações resistentes. O estado fisiológico da fêmea provavelmente também causa uma resposta individual. Em geral, os mosquitos não alimentados são maıs irritáveis pelo DDT do que os mosquitos alimentados (WHO 1975) .

Se uma população de mosquitos susceptível ê altamente irritada ou repelida por DDT, existe a possibilidade de haver o desenvolvimento de uma resistência comportamental. Isto foi definido como a "habilidade dos insetos, através de hábitos protetores ou comportamentais, de evitar contato letal com um tóxico" (Hess, 1952, apud Perry \& Agosin, 1974) Em laboratório, tal resistência comportamental foi demonstrada por Gerold \& Laarman (1967) que selecionaram Anopheles atropavus por 32 gerações, com base em seu comportamento de escape dos compartimentos com DDT. Eles descobriram que a irritação pelo DDT acarretava um aumento na atividade do vôo, mas a habilidade de escapar, em si, era um componente separado do comportamento. No campo, entretanto, tal fenômeno ainda não foi claramente demonstrado. Supõe-se que geralmente o aumento da exofilia no mosquito seja o resultado de um efeito seletivo do tóxico (Hamon et al., 1970) .

Observações preliminares (Hayes \& Charlwood 1977) indicam que o Anopheles darlingi Root, o vetor da malária nesta região, tende a evitar as superfícies borrifadas com DDT.

Este trabalho relata os resultados de testes usando uma caixa de excito-repelência, fornecendo dados quantitativos para A. darlingi, A. nuneztovari Gabaldon e Culex pipiens quinquefasciatus Say (= fatigans). Descrevese também o uso de tais caixas-testes como armadilhas de repouso para C.p. quinquefasciatus.

\section{MÉTodos}

As caixas-testes foram semelhantes àquelas usadas por Rachou et. al. (1973) As caixas são de $50 \mathrm{~cm}$ de cada lado, e foram feitas de madeira compensada de $15 \mathrm{~mm}$ de espessura. Elas têm duas aberturas: uma na parte su-

(•) - Instituto Nacional de Pesquisas da Amazônia, Manaus. 
perior e outra em uma dos lados. Estas podiam ser fechadas através de uma armadilha escorregadiça de fuga. Esta armadilha é uma gaiola telada, tendo um dos lados com $30 \mathrm{~cm}$. Um dos lados foi convertido em funil de compensado (Fig. 1). Os lados internos e o topo interno das caixas foram revestidos com pape! filtro branco. O chão não foi revestido, uma vez que se sabe bem que os mosquitos, e em particular os Anofelinos, normalmente não repousam no fundo de gaiolas, ou seja, em superficies horizontais (Rachou et al., 1973). Uma série de papéis foi borrifada por um funcionário da SUCAM, numa proporção de aproximadamente 2 gramas de DDT em pó umedecível por metro quadrado. Esta concentração é a mesma usada nos locais da campanha de borrifação de casas. A caixa revestida com o papel borrifado serviu como controle. O processo para cada teste foi o seguinte: um número conhecido de mosquitos foi suavemente soprado para dentro de cada caixa, através de aspiradores. O número de insetos usados para cada teste foi determinado pela disponibilidade dos mesmos. Foram usados $30 \mathrm{em}$ cada cai$x a$ nos testes usando A. nuneztovari e 50 ou 60 por caixa para cada teste com C.p. quinquefasciatus. Nos 5 testes com A. darlingi, dois tinham 70 , dois tinham 45 , em cada caixa. Devido à escassez de mosquitos no último teste, foram usados 33 no controle, e no teste 46 . As caixas foram mantidas na sombra, e o nivel de umidade foi mantido alto dentro das caixas, usando-se um chumaço de lã de algodão colocado no chão de cada uma antes de começar o teste. Todos os testes tiveram lugar na mesma hora do dia (entre 10 e 12 horas da manhã), e durante os testes a temperatura foi de aproximadamente $27^{\circ} \mathrm{C}$, e a umidade relativa foi de $70-80 \%$.

Depois de um período de aclimatação de 5 minutos, os insetos que tinham voado para dentro da armadilha de fuga foram coletados e contados. Os mosquitos não alimentados foram testados por 2 horas. Os C.p. quinquefasciatus alimentados com açúcar e os alimentados com sangue foram testados por 1 hora somente. No final do teste, o número de mosquitos sobreviventes que ficou dentro das caixas foi marcado.
As fêmeas dos Anofelinos não alimentadas foram coletadas na noite anterior. Elas foram usadas nos testes, uma vez que se constituiam no material disponível.

Os C. p. quinquefasciatus capturados enquanto repousavam dentro das casas, foram aiimentados com sangue antes dos testes. Os c. p. quinquefasciatus alimentados com açúcar e os não alimentados foram criados em labcratório. Quando foram testados, eram muliparos e com 3 a 7 dias de idade.

Quando usadas como armadilhas de repouso, as caixas foram colocadas numa casa, onde a população local de mosquitos Culex era alta. As aberturas das caixas foram expostas, afastando-se as gaiolas de topo para um dos lados, através de um deslizador, e somente foram fechadas por ocasião da remoção dos mosquitos. Os mosquitos que já estavam na armadilha de fuga do lado, foram coletados e considerados como sendo "fora"; os mosquitos que ficaram foram estimulados a voar para dentro das armadilhas de fuga, fazendo-se um leve distúrbio nas paredes da caixa. Estes foram coletados e considerados como "dentro". Quando mais nenhum mosquito voou para dentro das armadiIhas, a armadilha de topo foi removida, e os interiores das caixas foram examinados para verificar se havia mais algum mosquito. Se algum tivesse ficado, teria sido contado e removido com um aspirador.

Foi evidente no começo a possibilidade de que a posição das caixas influenciaria a intensidade da captura. Portanto, todo dia as posições relativas das caixas foram invertidas, e os resultados obtidos foram tidos como pares diários, para que o efeitơ da posição fosse reduzído.

Foram feitos testes de susceptibilidade ao DDT usando o teste padrão da OMS para mosquitos adultos.

\section{RESUltados}

Os resultados para as duas espécies anofelinos são apresentados na Tabela 1 e no Gráfico 1. Foram feitos 14 testes, usando um total de 832 A. nuneztovari e 5 testes usando 539 A. darlingi. Pode-se notar que ambas as espécies evitam as superfícies borrifadas com DDT, 

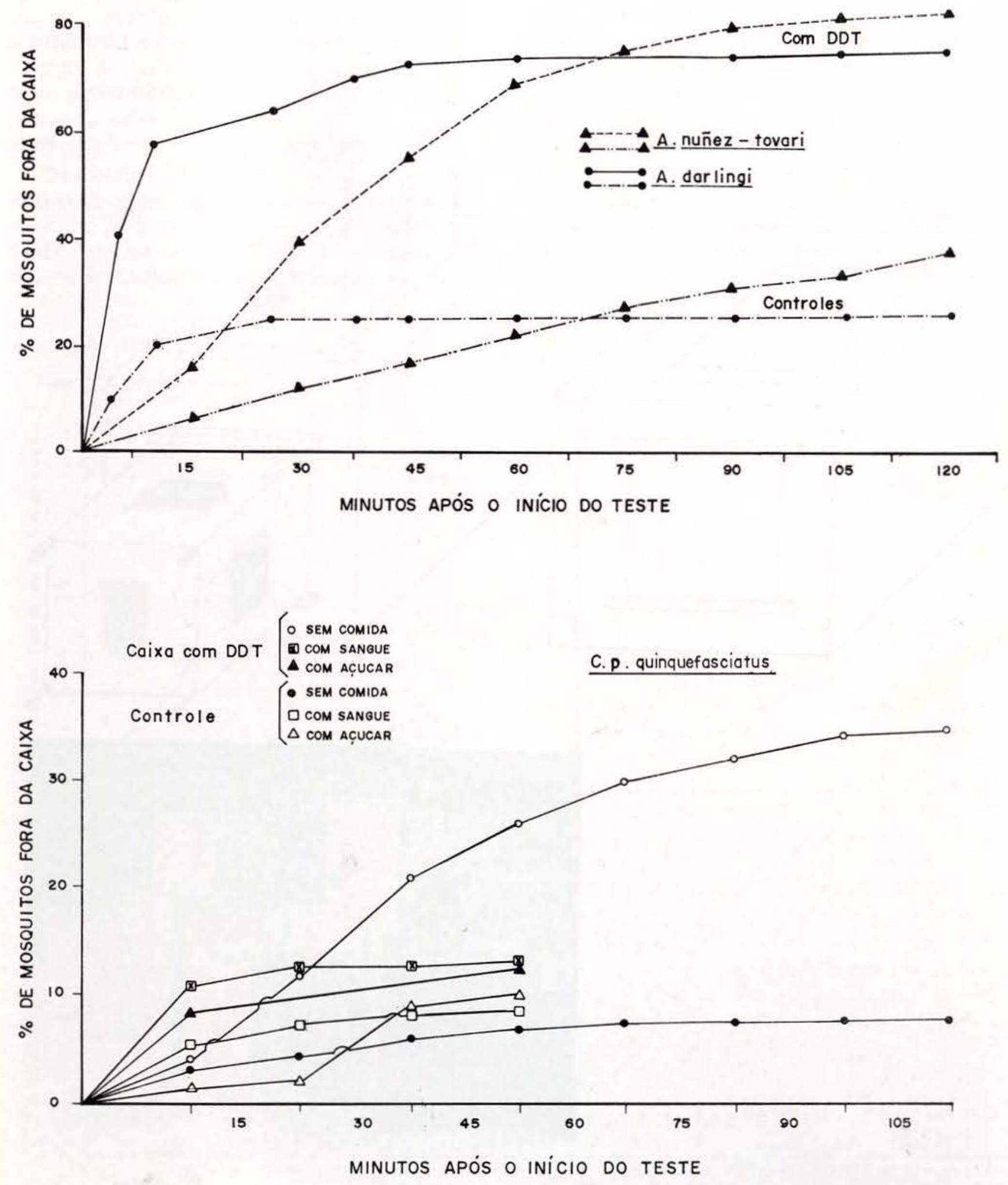

Gráfico I - Curvas representativas da porcentagem de mosquitos que sairam das caixas

o uso de caixas... 

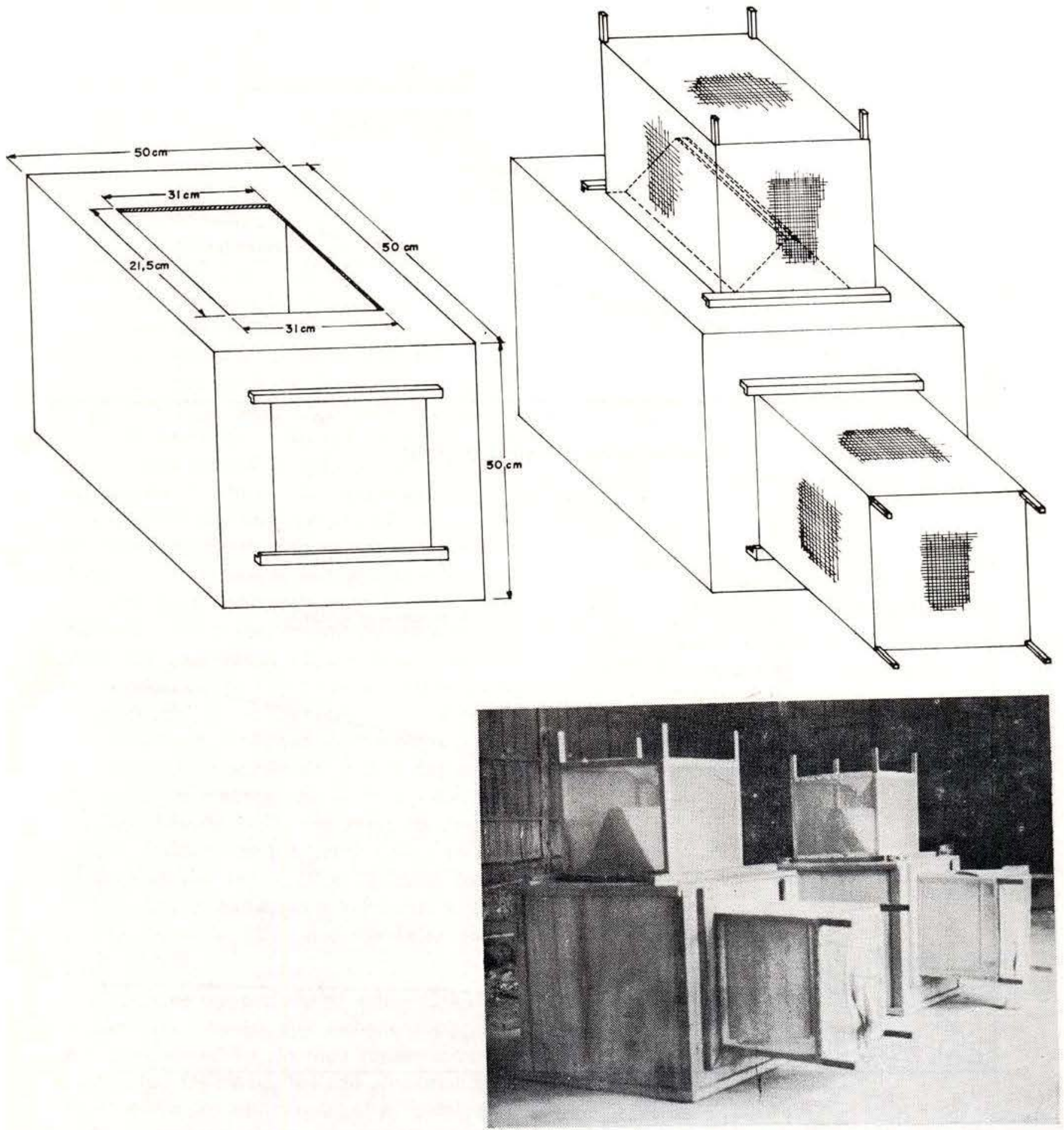

Fig. 1 - Esquema das caixas usadas nos testes e a posição das mesmas durante o decorrer dos experimentos 
mas que esta resposta foi mais pronunciada nos primeiros 15 minutos de cada teste, no que diz respeito ao $A$. darlingi, ao passo que a resposta foi mais gradual no $A$. nuneztovari testado. Não houve uma diferença significativa entre a percentagem de $A$. nuneztovari que escapou da caixa teste entre o $1^{0}$ e o último teste.

Descobriu-se que a população de $A$. darlingi testada é susceptível ao DDT, usando equipamento para testes de susceptibilidade da OMS. Isto foi reforçado pelo fato de que todos os $A$. darlingi que não escaparam da caixa de DDT foram encontrados mortos, duas horas após o início do teste. Uma limitaçăo no número de $A$. nuneztovari impediu o uso do equipamento para esta espécie. Porém, o mosquito nesta região não entra em contato com o inseticida, pelo fato de que ele não penetra em casas, e também de que não há borrifação na agricultura nesta região. Entretanto, é improvável que seja resistente ao DDT.

A população local do C. p. quinquefasciatus é, portanto, muito resistente ao DDT. Quinze fêmeas foram observadas em 3 ocasiões, por 18 horas, em contato constante com $4 \%$ de
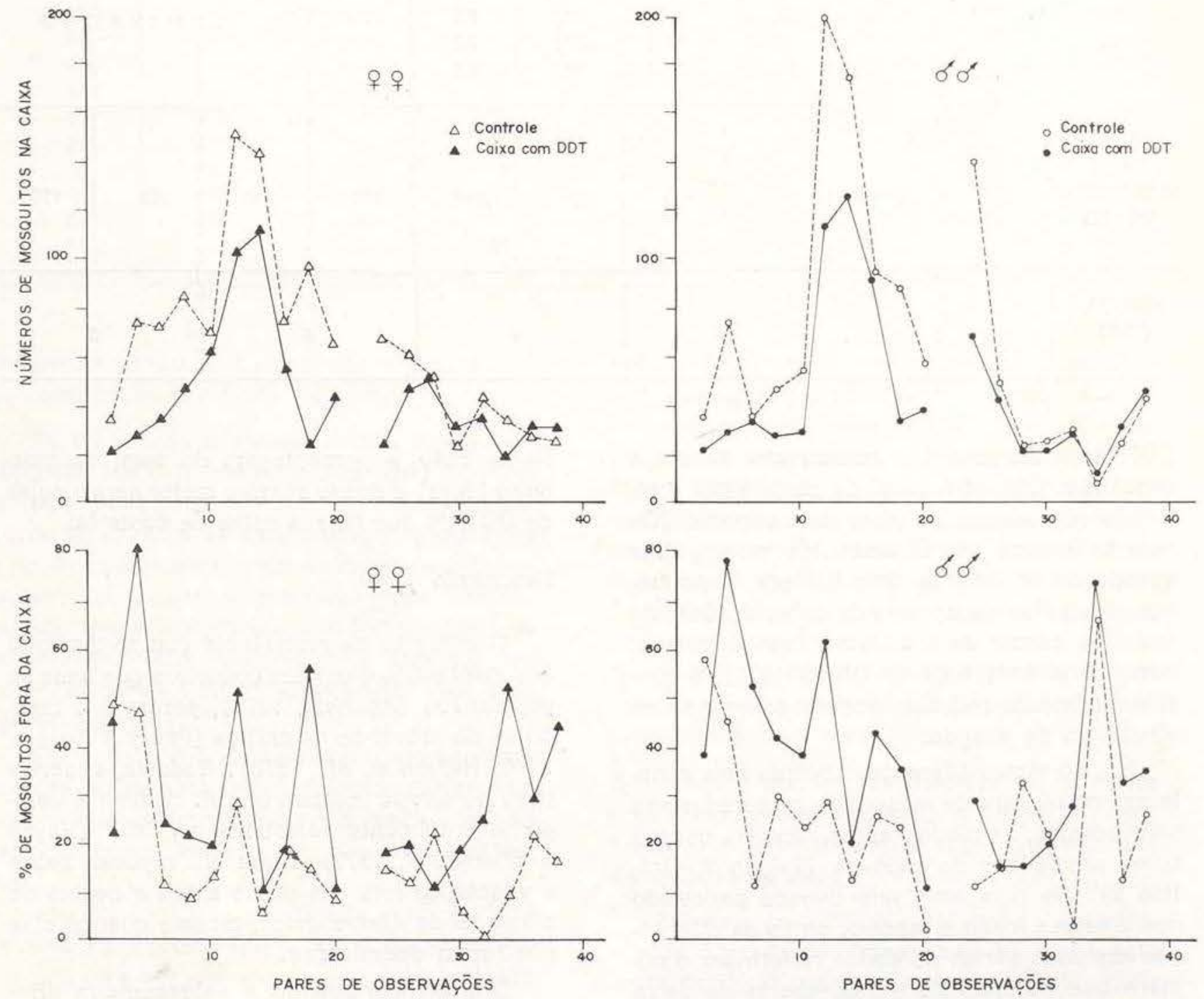

Gráfico II - Total de C. p. quiquefasciatus coletada nas armadilhas de repouso (pares de observações) e a porcentagem "FORA" 
TABELA 1 - Resultados dos Testes de Excito-Repelencia

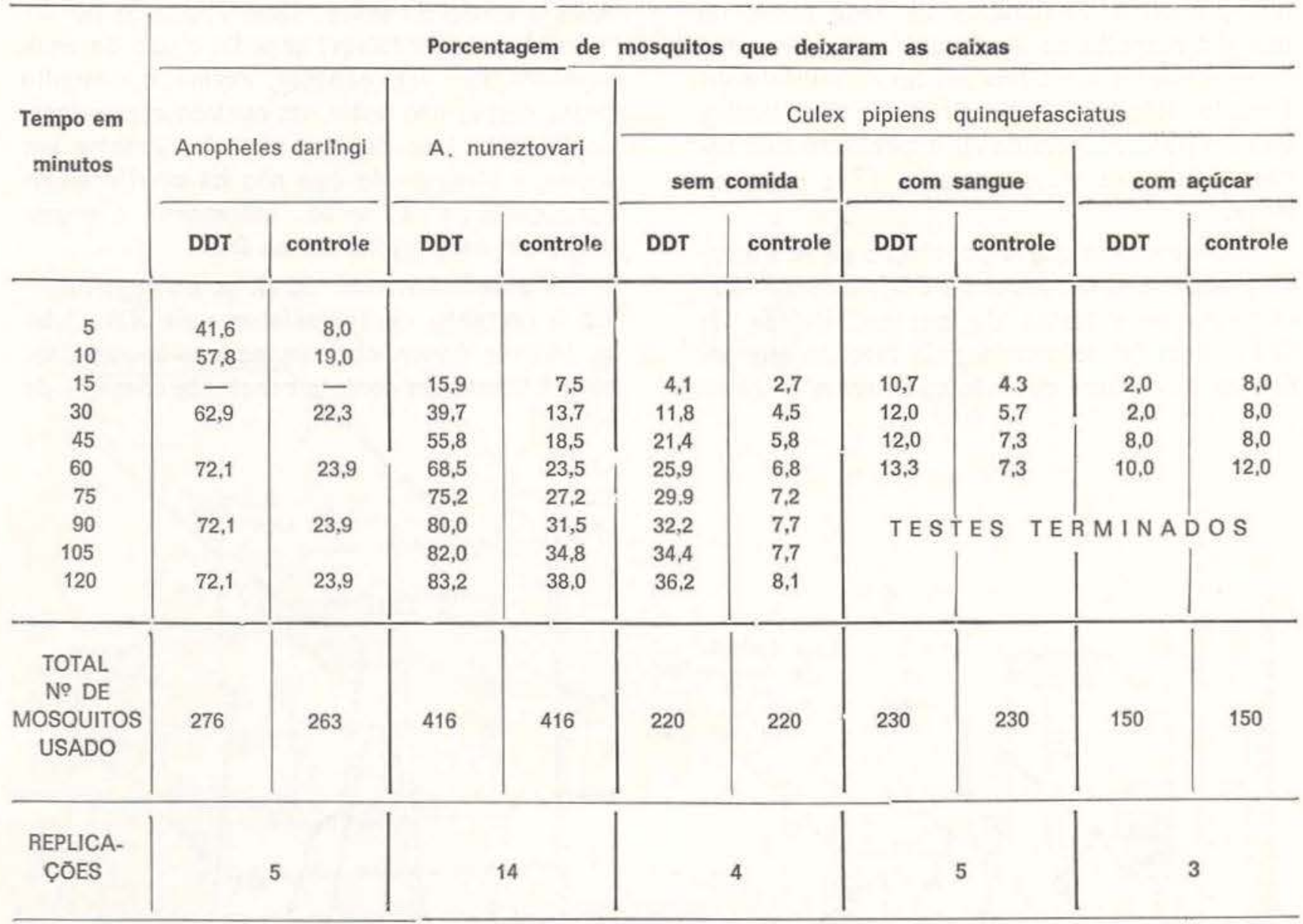

DDT, e nenhuma delas morreu nas 24 horas seguintes. Este alto nível de resistência é refletido nos resultados para esta espécie (Tabela 1, Gráfico 1). O inseticida mostra uma quantidade mínima de irritabilidade, com menos mosquitos escapando da caixa de DDT do que nas caixas de controle. Esta diferença, como se poderia esperar (WHO 1975), é consideravelmente reduzida quando os insetos se alimentam de sangue.

Há, portanto, diferenças ligeiras mas constantes no número de mosquitos capturados nas caixas-testes, quando estas foram usadas como armadilhas de repouso (Tabela 2, Gráfico 2). Se o número que tivesse penetrado nas 2 caixas fosse o mesmo, então as diferenças nas duas séries de dados refletiriam o número que escapou da tampa aberta da caixa de DDT. Estă tendência de escapar é refletida na porcentagem do total de capturas feitas na armadilha de fuga, situada no lado da caixa.
Neste caso, a porcentagem do total de mosquito "fora" é quase sempre maior para a caixa de DDT do que para a caixa de controle.

\section{DISCUSSÃo}

O conceito de resistência comportamental aos inseticidas é um dos conceitos que mais se popularizou (Agarwai, 1978), porém é o mais difícil de provar-se na prática (Peery \& Agosin, 1974; Hamon et al., 1970). Todavia, observações no campo indicam que $A$. darlingi é comportamentalmente resistente ao DDT (Hayes \& Charlwood, 1977) e que ele repousa sobre a vegetação fora das casas antes e depois de alimentar-se dentro delas, mesmo quando elas não foram dedetizadas.

Este comportamento é sobremaneira diferente do observado por Shannon (1933), que coletou "uma população diurna de $A$. darlingi que repousava dentro das casas" e, pode-se 


\begin{tabular}{|c|c|c|c|c|c|c|c|}
\hline \multicolumn{4}{|c|}{ MACHOS } & \multicolumn{4}{|c|}{ FE MEA S } \\
\hline \multicolumn{2}{|c|}{ Caixa com DDT } & \multicolumn{2}{|c|}{ Controle } & \multicolumn{2}{|c|}{ Caixa com DDT } & \multicolumn{2}{|c|}{ Controle } \\
\hline $\begin{array}{l}\text { Total } \\
\text { coletado }\end{array}$ & $\%$ fora & $\begin{array}{c}\text { Total } \\
\text { coletado }\end{array}$ & $\%$ fora & $\begin{array}{c}\text { Total } \\
\text { coletado }\end{array}$ & $\%$ fora & $\begin{array}{c}\text { Total } \\
\text { coletado }\end{array}$ & $\%$ fora \\
\hline 21 & 38 & 36 & 58 & 22 & 45 & 35 & 49 \\
\hline 28 & 79 & 74 & 45 & 26 & 81 & 76 & 47 \\
\hline 33 & 52 & 36 & 11 & 36 & 24 & 72 & 11 \\
\hline 27 & 41 & 47 & 30 & 49 & 22 & 85 & 8 \\
\hline 29 & 38 & 52 & 23 & 62 & 19 & 70 & 13 \\
\hline 114 & 61 & 201 & 28 & 102 & 51 & 152 & 29 \\
\hline 127 & 20 & 175 & 12 & 113 & 8 & 142 & 6 \\
\hline 94 & 43 & 94 & 26 & 76 & 18 & 74 & 19 \\
\hline 31 & 35 & 89 & 24 & 25 & 56 & 97 & 15 \\
\hline 36 & 11 & 58 & 3 & 41 & 10 & 65 & 9 \\
\hline- & - & - & - & - & - & - & - \\
\hline 69 & 29 & 140 & 11 & 23 & 17 & 66 & 15 \\
\hline 41 & 15 & 47 & 15 & 48 & 19 & 60 & 13 \\
\hline 22 & 14 & 24 & 33 & 52 & 10 & 52 & 23 \\
\hline 21 & 19 & 26 & 23 & 32 & 19 & 21 & 5 \\
\hline 30 & 27 & 30 & 3 & 38 & 24 & 42 & 0 \\
\hline 11 & 73 & 9 & 67 & 20 & 52 & 33 & 9 \\
\hline 34 & 32 & 26 & 12 & 35 & 29 & 28 & 21 \\
\hline 44 & 55 & 42 & 26 & 32 & 44 & 25 & 16 \\
\hline
\end{tabular}

deduzir então que tenha ocorrido uma mudança no comportamento deste mosquito.

Se de fato a resistência existe, então uma resposta mais intensificada ao DDT deveria ter sido antecipada. Portanto, a resposta rápida do $A$. darlingi às caixas-testes, comparada a do A. nuneztovari, e ao fisiologicamente resistente C. p. quinquefasciatus pode ser um reflexo desta resistência comportamental. A porcentagem que escapou pode também ser uma função da irritabilidade básica das diferentes espécies.

Portanto, a fim de se obter maiores informações sobre este fator, são necessárias três coisas que diferenciam as linhagens comportamentalmente resistentes das não-resistentes:

1 - Papéis com uma quantidade exata de DDT deveriam ser produzidos e utilizados de tal forma que uma série graduada de testes pudesse ser feita;

2 - Testes excito-repelentes precisariam ser padronizados, como são os testes fisiológicos
(WHO 1975); a porcentagem de mosquitos que escapa nos primeiros 15 minutos nas diferentes concentraçōes de DDT seria um teste tão efetivo como qualquer outro. Se a porcentagem que escapa das caixas é maior nas concentrações mais altas de DDT pode ser possível de se definir resistências comportamentais. Isto é quando uma grande porcentagem (diferença significativa) escapa das caixas de testes nas concentrações mais baixas do que nesta que vai dar um CL 50 (conto letal 50 ), usando o teste normal da OMS para ver a sus. ceptibilidade aos inseticidas;

3 - Finalmente, em se tratando de mosquito criado em laboratório, seria necessário determinar se a resposta existe como um resultado de seleção ou não.

Em uma área como esta, onde a resistência do parasita às drogas anti-maláricas está se tornando difundida (Ferraroni \& Waki, 1977; Bruce-Chawatt, 1970), a possibilidade de uma "resistência comportamental" pode acarretar graves ocorrências. 


\section{AGRADECIMENTOS}

Os autores agradecem à SUCAM pela colaboração prestada no decorrer deste trabalho. - Durante este mesmo trabalho, Norival D. Paraluppi recebeu bolsa de estudos do ConseIho Nacional de Pesquisas.

- Agradecemos à Sra. Maria Regina Filgueiras dos Reis pela tradução e também ao $\mathrm{Dr}$. Jorge Arias pelas críticas construtivas.

\section{SUMMARY}

Excito-repellancy boxes were used to test the effect of DDT on populations of Anopheles darlingi, A. nuneztovari and Culex pipiens quinquefasciatus from in and around the city of Manaus, Amazonas. The two Anopheline species showed marked escape responses whereas the very resistant Culex population was little affected. The test boxes were also used as resting traps for C. P. quinquefasciatus and in this case slight differences 'between 'the DDT' sprayed box and an unsprayed control were evident. The need to standardize excito-repellancy testes' in order to clarify the concept of behavioural resistance is discussed.

\section{BIBLIOGRAFIA CITADA}

AgARWALL, A

1978 - Malaria makes a comeback. New Scientist, $77(1088)$ : 274-277. ,

BRUCE-CHWATt, L. J.

1970 - Global review of malaria control and eradication by attack on the vector. Misc. pub. ent. soc. ameri., $7: 7-24$.
FERRARONI, J. J. \& WAKI, S.

1977 - Resistência do Plasmodium às Chloroquinas no Estado do Amazonas, detectada pelo método in vitro. Acta Amazonica, 7(1): 147-148.

Gerold, J. L. \& LaArman, J. J.

1967 - Behavioural responses to contact with DDT in Anopheles atroparvus Nature, 215: 518-520.

Hamon, J.; Mouchet, J.; Brenques, J. \& Chauvet, G.

1970 - Vector ecology and behaviour before, during and after application of control measures. Misc. pub. ent. soc Amer., $7: 28-41$.

Hayes, J. \& Charlwood, J. D.

1977 - O Anopheles darlingi evita DDT numa área de malaria resistente a drogas. Acta Amazonica, $7(2): 289$.

Hess, A. D.

1952 - Amer. J. Trod. Med. Hyg., 1: 371.

PERry, A. S. \& Agosin, A.

1974 - The Physiology of insecticide resistence by insects. In: The Physiology of the insecta. - Rockstein, Morris, ed. Academic press. v. $6, p \cdot 3-102$.

Rachou, R. G.; Schinazi, L. A. \& Moura Lima, M.

1973 - An intensive study of the causes for the failure of residual DDT Spraying to interrupt the transmission of malaria in Atalaya and Falla, two villages on the coastal plain of El Salvador, Central America. Revta. bras. Malar. Doenç. Trop., 25(1-4) : 5-293.

SchOOF, H. F.

1970 - Physiological resistance and development of resistance in Field populations. Misc. Pub. Ent. Soc. Amer., 7: 45-61.

SHANNON, R. C.

1933 - Anophelines of the Amazon Valley. Proc. Ent. Soc. Wash., 35(7) : 121-143.

WHO (World Health Organization)

1975 - Manual on practical entomology in malaria. Part II. WHO offset publication, 13: 1-186.

(Aceito para publicação em 18/08/78) 